\title{
QoE-Aware Device-to-Device Multimedia Communications
}

\author{
Liang Zhou \\ Nanjing University of Posts and Telecommunications, China \\ liang.zhou@njupt.edu.cn
}

\begin{abstract}
Multimedia services over mobile device-to-device (D2D) networks has recently received considerable attention. In this scenario, each device is equipped with a cellular communication interface, as well as a D2D interface over a shared medium. In this work, we study the performance properties of the mobile D2D communications in the framework of user satisfaction, and develop a fully distributed QoE-aware multimedia communication scheme (QAMCS). Specifically, we translate the opportunistic multimedia communications issue into a stochastic optimization problem, which opens up a new degree of performance to exploit. Moreover, QAMCS is designed for a heterogeneous and dynamic environmen$\mathrm{t}$, in which user demand, device mobility, and transmission fashion may vary across different devices and applications. Importantly, QAMCS is able to maximize the user satisfaction and only needs each device to implement its own scheme individually in the absence of a central controller.
\end{abstract}

\section{Categories and Subject Descriptors}

H.4 [Information Systems Applications]: Miscellaneous; C.2 [Computer-Communication Networks]: Distributed Systems

\section{General Terms}

Design, performance

\section{Keywords}

D2D communications; mobile networks; multimedia communications; QoE

\section{INTRODUCTION}

Recently, there has been a tremendous increase in the use of smart devices, e.g., smart phone, tablet, etc. These devices are usually equipped with two wireless communication interfaces, that is, long-range cellular communication and shortrange device-to-device (D2D) communication interfaces. In particular, D2D communications can enable each device to communicate with each other without the help of base station. Therefore, it provides a platform for high data rate transmission. Simultaneously, there has been an explosion in multimedia communications, and it is expected that massive multimedia services over D2D networks will play a dominant role in the $5 \mathrm{G}$ mobile communications $[1-3]$.

Meanwhile, quality of experience (QoE)-aware multimedia communication has received significant attention recently [4]. In contrast to traditional QoS transmission which only concerns about the communication parameters, e.g., bandwidth, jitter, delay, QoE pay more attention to the subjective measurement of end-to-end multimedia service, from the userąrís point of view $[5,6]$. Usually, QoE is measured by mean opinion score (MOS) which reflects the degree of user satisfaction from a scale of 1 (unacceptable) to 4.5 (excellent) [4].

Essentially, addressing QoE-aware D2D multimedia communication is challenging for a variety of reasons, and the most critical ones are the subjective measurement and opportunistic communication. Firstly, different users usually have diverse multimedia content demands, and even for the same multimedia service, its priority level for different users may be different as well, and the QoE of each user is usually distinct accordingly. Secondly, devices follow various and random mobility directions and velocities, thereby having opportunistic data transmission probability. All the above challenges principally determine the subjective communication quality or user satisfaction. In this sense, a QoE-aware multimedia transmission policy should be elaborately designed to accommodate the above technical challenges.

To the best of our knowledge, QoE-aware multimedia transmission in D2D networks has not been directly considered in the communications literature. Specifically, the related works, e.g., $[5,7,8]$, are only applicable for peer-to-peer networks by employing various models to analyze the system capacity. However, the user demand, multimedia content, and user satisfaction model are either known parameters or unknown constants in existing works, which obviously 
are unpractical in many communication scenarios [4]. Thus, their problems are orthogonal to the main focus of this paper in which the user demand, QoE model, device mobility, and transmission fashion are totally unknown. Importantly, we extend existing data dissemination schemes in two critical aspects.

- We formulate the distributed multimedia dissemination problem based on real observed data popularity and priority, and investigate a general mobile D2D system with subjective measurement and opportunistic communication.

- We dynamically set D2D transmission range and D2D communication time in a precisely mathematical manner, and derive the optimal performance bounds for the proposed scheme.

The rest of the paper is organized as follows. Section II describes the considered mobile D2D networks. In Section III, we summarize our main results on the performance properties and data dissemination scheme. Rigorous proofs are presented in Sections IV. Subsequently, Section V provides numerical simulation results and Section VI concludes the paper with a summary.

\section{SYSTEM MODEL}

There are $\mathcal{U}=\{1, \ldots, U\}$ devices, and the transmission range and mobility velocity of device $u(u \in \mathcal{U})$ are denoted by $R_{u}$ and $V_{u}$, respectively. To depict a general device mobility, we employ the random walk mobility model introduced in [5]. Basically, the mechanism of D2D multimedia communications is described as follows: when device $u$ demands a new multimedia service, $u$ first requests the application availability from its neighbors, locating within its D2D transmission range $R_{u}$, for some time (called D2D time). If the application can be provided by the neighbor devices, the nearest one $j$ will transmit the application to $u$ via D2D communications. If not, device $u$ will increase the transmission range $R_{u}$ and mobility velocity $V_{u}$ accordingly. Once the multimedia application request can not be satisfied during the D2D time, device $u$ utilizes the cellular communications to get the multimedia services.

Usually, $R_{u}$ and $V_{u}$ are constants in existing literature while they are dynamic parameters in this work. In a practical D2D communication scenario, $R_{u}$ and $V_{u}$ can be dynamically set to maximize the user QoE. Intuitively, improving $R_{u}$ and $V_{u}$ can lead to a higher communication D2D probability, but at the cost of more energy cost. Typically, the total energy cost for device $u$, denoted by $E_{u}$, can be modeled as:

$$
E_{u}=C_{r} \times R_{u}+C_{v} \times V_{u},
$$

where $C_{r}(\mathrm{~J} / \mathrm{m})$ and $C_{v}(\mathrm{~J} / \mathrm{m} / \mathrm{s})$ are energy cost constants.

There are $\mathcal{F}=\{1, \ldots, F\}$ different kinds of multimedia applications in all the devices' caches, for example, real-time video, online game, audio, etc. We define $P_{u, f}=1$ if device $u \in \mathcal{U}$ is able to provide application $f \in \mathcal{F}$, and $P_{u, f}=0$ otherwise. The matrix $\mathcal{P}=\left(P_{u, f}\right)_{u \in \mathcal{U}, f \in \mathcal{F}}$ represents the application distribution of in all the devices. Moreover, we denote the number of application $f$ by $P_{f}=\sum_{u \in \mathcal{U}} P_{u, f}$, and the application distribution of $u$ by $P_{u}=\sum_{f \in \mathcal{F}} P_{u, f}$. Due to the random contact of D2D communication, we assume that device $u \in \mathcal{U}$ communicate with device $j \in \mathcal{U}$ according to a Poisson process with rate $\omega_{u, j}$ which is obviously strongly related to $R_{u}$ and $V_{u}$. In this case, the communication probability of the $\mathrm{D} 2 \mathrm{D}$ communication can be written as $\omega_{u, j}\left(R_{u}, V_{u}\right)$. It should be note that compared to traditional D2D communication, we have the following characteristics:

1. Without the help of the base-station. In our model, base station does not provide any information on data and control. All the information collection and application services are provided by the individual devices within the D2D time.

2. $R_{u}$ and $V_{u}$ are dynamic parameters, and can be set by the individual devices according to the need of the application service.

3. Blind transmission. All the system state, e.g., application distribution, demand model, etc., are unknown for the devices, and the devices should learn from the local contact to estimate the global information.

To design a flexible QoE model to capture the real user satisfaction on D2D multimedia service, we should jointly take into account the user gain and cost. In terms of the user gain from the multimedia service, it is well-known that user gain is very sensitive to the waiting time $\tau$, which is formally defined as the elapsed time between the application demand and service complement. Typically, the gain function $G_{f}(\tau)$ is a non-increasing function of the $\tau$. According to the characteristics of different multimedia applications, we can roughly divide them into three main categories: emergent, normal, and tolerant model. In particular, emergent model usually employs the format of $G_{f}(\tau)=\tau^{-1}$, normal model is represented by $G_{f}(\tau)=e^{-\tau}$, and the tolerant model is designed as $G_{f}(\tau)=(\sqrt{\tau})^{-1}$. In terms of the cost function $F$, it is directly related to two parts: energy cost $E_{u}$ and communication cost $C_{u}$, that is, $F_{f}\left(E_{u}, C_{u}\right)$.

We define $W_{u, f}$ to be the expected user satisfaction generated by a request for file $f$ from user $u$, and obviously this value is directly related to the QoE model. For D2D communications, we can formally define $W_{u, f}$ based on the definition of $\omega_{u, j}\left(R_{u}, V_{u}\right)$, by taking into account the gain and cost, we can design an appropriate function $W_{u, f}$ based on its definition. Specifically, we have (2), in which the expected gain and cost are compared and the possible resolution formation are provided. where $G_{f}^{\prime}$ and $C_{f}^{\prime}$ are the first order derivatives of the function.

To maximize the social welfare, we seek to optimize the Total User Satisfaction (TUS) $W(\mathcal{P})$ for all the devices and the applications

$$
\begin{array}{cc}
\max & W(\mathcal{P})=\sum_{f \in \mathcal{F}} \sum_{u \in \mathcal{U}} W_{u, f}(\mathcal{P}) \\
\text { s.t. } & \sum_{f \in \mathcal{F}} P_{u, f} \leq S_{u} \\
& P_{u, f} \in\{0,1\}, \forall u \in \mathcal{U}, \forall f \in \mathcal{F} .
\end{array}
$$

Intuitively, TUS is determined by a number of parameter$\mathrm{s}$, including the transmission range, mobility velocity, D2D 


$$
W_{u, f}=G_{f}(0)-\left(1-P_{u, f}\right) \int_{0}^{\tau} \exp \left(-t \sum_{u \in U} P_{u, f} \omega_{u, j}\left(R_{u}, V_{u}\right)\right)\left(\mathrm{G}_{f}^{\prime}(t)-F_{f}^{\prime}\left(\mathrm{E}_{u}(t), \mathrm{C}_{u}(t)\right)\right) d t
$$

time, application popularity, as well as the application priority. As a result, (3) is a complex multimedia communication problem which is coupled with data storage and transmission strategies.

\section{MAIN RESULTS}

In this work, we consider a general mobile D2D communication scenario with average mobility velocity $\bar{V}$ and transmission range $\bar{R}$. The main results are formally stated in this section, and the rigorous proofs are provided in Section 4.

\subsection{Performance Properties}

TheOREM 1. For a general mobile D2D system with $U$ devices, for each device $u$, assume the average transmission range $\bar{R}$ and a class of mobility velocity $V_{u}$ with finite mean $\mu(\mu \geq 0)$ and variance $\sigma^{2}\left(\sigma^{2} \geq 0\right)$. For any distributed data dissemination scheme $\mathcal{P}$, the expected value of TUS, $\mathbb{E}[W(\mathcal{P})]$, satisfies $^{1}$

$$
\mathbb{E}[W(\mathcal{P})] \leq \Theta\left(U \cdot \exp \left(-\frac{\left(\sigma^{2}+1\right)}{\bar{R} U \mu}\right)\right) .
$$

It is shown in Theorem 1 that, for any distributed scheme, the achievable performance depends on the number of devices, transmission range, and mobility velocity. From the system's perspective, this theorem exhibits the benefits of the mobile D2D communications since large mobility velocity yields high TUS.

Moreover, we investigate the convergence rate in terms of asymptotical convergence rate (ACR) $\gamma$ introduced in $[9$, Definition 2]. In particular, we have:

THEOREM 2. Under the same conditions of Theorem 1, the asymptotical convergence rate $\gamma$ for any distributed data dissemination satisfies

$$
\gamma \leq O\left(\exp \left(-\frac{\sigma^{2} U t}{2 \bar{R} \mu}\right)\right)
$$

Theorem 2 establishes a fundamental ACR limit of various distributed schemes. In particular, the device mobility and network scale always affect the convergence rate.

\subsection{QoE-Aware Data Dissemination Scheme}

In order to attain the performance bounds shown in Theorem 1 and Theorem 2, the most challenging issue lies in how to translate the objective function (3) into a stochastic optimization problem (recall that each device communicates with others in a random manner). In fact, it is not a easy

\footnotetext{
$\overline{{ }^{1} \text { In terms of two functions } x(n)}$ and $y(n), x(n)=O(y(n))$ indicates that $|x(n) / y(n)|$ remains bounded as $n \rightarrow \infty, x(n)=$ $\Theta(y(n))$ shows that $x(n)=O(y(n))$ and $y(n)=O(x(n))$, and $x(n)=o(y(n))$ denotes that $|x(n) / y(n)| \rightarrow 0$ as $n \rightarrow \infty$.
}

task due to the following reasons: 1) the goal function is not a regular convex or concave function since $R_{u}$ and $V_{u}$ are dynamic parameters, 2) the relationship between the variables are not clear, thus the goal function cannot be divided into sub-functions separately, and 3) how to construct the space variables to describe the dynamics of $\mathrm{D} 2 \mathrm{D}$ time is not clear either. As a result, a very important issue for the mobile D2D system is that dissemination policy implicitly adapts to current application distribution, cache update, and demand prediction without an explicit central controller for the information collection of the application popularity or priority. In this work, we propose a QoE-aware multimedia communication scheme (QAMCS), which is presented in Algorithm I.

Essentially, the underlying logic of QAMCS builds on the following facts: 1) the global application popularity and priority can be estimated from the local contact with each devices, 2) the user demand, although it is random, can be predicted in a stochastic sense, and 3) the variables of the goal function can be simplified when $R_{u}$ and $V_{u}$ are averaged with a certain period of time. As a result, we can employ the opportunistic study processing to realize the stochastic optimization. In this case, the core problem is to properly set transmission range $R_{u}$, mobility velocity $V_{u}$, D2D time function $\chi$, popularity function $\phi$, and priority function $\varphi$ to improve the estimation accuracy as much as possible.

We first consider the relationship between the waiting time and the transmission fashion. Mathematically, it is a classical multi-variable interaction problem, since both imply a number of variables. Specifically, the waiting time heavily depends on the transmission fashion, while the transmission fashion is also strongly related to the waiting time. By applying opportunistic study method to analyze the availability of the request file and the impact of waiting time on TUS, we explicitly derive the functions of the D2D time. Then, we use the stochastic optimization method to jointly achieve the priority and popularity functions, which are listed in Table 1. Using these results, QAMCS only requires each user to compute its multimedia communication policy individually, without the knowledge of application popularity or communication opportunity. 


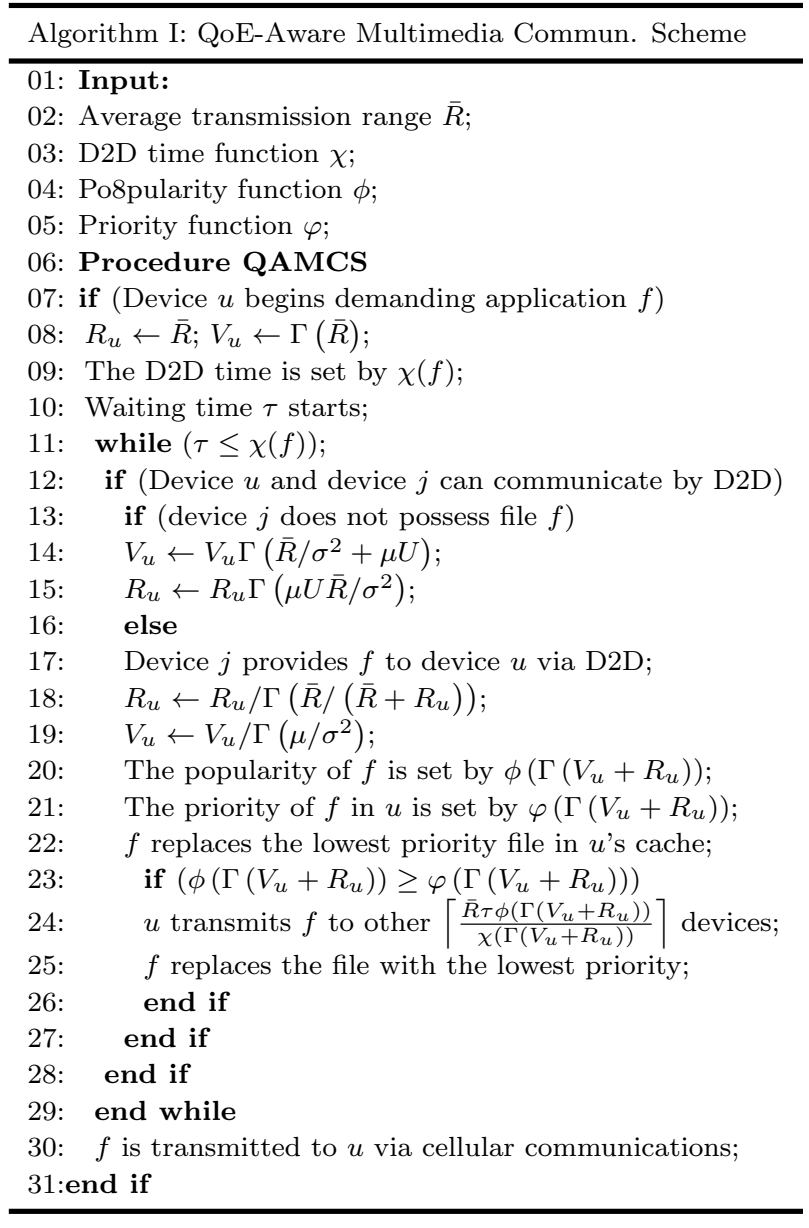

\section{PERFORMANCE ANALYSIS}

\subsection{Total User Satisfaction}

According to the above discussion, to decompose the goal function (3), it is necessary to clarify the following three basic facts: 1) the cache update process should follow the user demand and topology of the devices; 2) content priority is strongly related to the user demand and the availability of the content; 3) The transmission fashion should jointly consider the application popularity and priority. To realize the above goal, three detailed steps are designed as follows.

Step 1: Establishing the relationship between the application popularity with the steady application distribution.

Lemma 1. Let $\pi(t)$ be the stationary distribution of $\mathcal{P}(t)$, $\omega_{\text {avg }}(t)$ be the average value of $\omega_{u, j}(t)$ given any devices $u$ and $j$. Then,

$\mathbb{E}\left[\phi(\mathcal{P}(0)) \cdot \omega_{\text {avg }}(0)\right] \geq \max _{u} \phi\left(\sum_{f} P_{u, f}(0) \cdot \omega_{u, j}\left(R_{u} / V_{u}\right)\right)$.

Proof. Based on the definitions of $P_{u, f}$ and $\phi\left(\omega_{u, j}(t)\right)$, $\pi(t)$ can be set by:

$$
\begin{aligned}
\pi(t) & \propto \prod_{u} \frac{\exp \left(\sum_{f, j} \omega_{u, j}(t) P_{u, f}(t)\right)}{\sum_{j} \omega_{u, j}(t)} \\
& \propto \exp \left(\sum_{f} \sum_{j} \sum_{u}\left(\omega_{u, j}(t) P_{u, f}(t)-\log \omega_{u, j}(t)\right)\right) .
\end{aligned}
$$

To simplify the expression, we have

$$
G(\mathcal{P}) \triangleq \sum_{u} G_{u}(\mathcal{P}) \triangleq \sum_{u, j, f}\left(\omega_{u, j}(t) P_{u, f}(t)-\log \omega_{u, j}(t)\right) .
$$

Moreover, because the value of $\omega_{u, j}(t)$ is bounded for all $u$ and $j$, we have the following inequality

$$
0 \leq \sum_{u, j} \log \omega_{u, j}(t) \leq O\left(\sum_{u, j, f} \omega_{u, j}(t) P_{u, f}(t)\right) .
$$

In addition, due to the definition of $G(\mathcal{P})$, we have

$$
G(\mathcal{P}) \leq \sum_{u, j} \omega_{u, j}(t) P_{u}(t) \leq G(\mathcal{P})+O\left(\sum_{u, j} \log \omega_{u, j}\right) .
$$

Define $\bar{P}=\arg \max _{\mathcal{P}} \sum_{f} \sum_{u, j} \omega_{u, j}(t) P_{u, f}(t)$,

$$
\begin{aligned}
\mathbb{E}\left[\sum_{f, u, j} \omega_{u, j} P_{u, f}\right] & \geq \mathbb{E}[G(\mathcal{P})] \\
& \geq \max _{\mathcal{P}} G(\mathcal{P})-\sum_{f, u, j} \log \left(\omega_{u, j} P_{u, f}\right) \\
& \geq G(\bar{P})-\max _{j} \sum_{f, u} \log \left(\omega_{u, j} P_{u, f}\right) \\
& \geq \max _{\mathcal{P}} O\left(\log \omega_{u, j}(t)\right) .
\end{aligned}
$$

Next, we characterize the $\sum_{f, j, u} \omega_{u, j} P_{u, f}$. In particular, we study the form of $\left[\phi(\mathcal{P}(0)) \cdot \omega_{\text {avg }}(0)\right]$, in particular, we need to consider the performance of $\max _{u, f} \sum_{j} \omega_{u, j} P_{u, f}$, that is

$$
\phi(\mathcal{P}(0)) \cdot \omega_{\text {avg }}(0) \geq \sqrt{\max _{u, f} \sum_{j} \omega_{u, j} P_{u, f}} .
$$

With that, we can complete this proof.

Step 2: Estimating the steady distribution of $\pi(0)$.

The core problem right now is how to estimate the distribution of $\pi(0)$ based on the local observation in a relatively simple and practical method. By definition, $\pi(0)$ is related to the initial condition $\mathcal{P}(0)$. To this end, we consider the following Lyapunov function

$$
L(\mathcal{P}(t))=\sum_{u} \Phi\left(\sum_{j} \sum_{f} \omega_{u, j}(t) P_{u, f}(t)\right),
$$

where $\Phi(x)=\int_{0}^{x} \phi(y) d y$. To estimate $\pi(0)$, it is necessary to show that $\mathcal{P}(t)$ is close enough to that of $\pi(t)$ when 
Table 1: Different function expressions under different scenarios

\begin{tabular}{c||c|c|c}
\hline \hline & Emergent Model & Normal Model & Tolerate Model \\
\hline \hline $\bar{R}$ & $2 \sqrt{U \mu}$ & $\exp \left(U^{-1} \mu\right)$ & $\sqrt{U \mu}$ \\
\hline$\chi$ & $(2 U \mu+\bar{R}) / \sigma^{2}$ & $\left(\exp \left(U^{-1} \bar{R}\right) \sigma^{2}+\Gamma(U) \sigma^{2}\right) / \mu$ & $\left(\exp \left(U^{-1} \sigma^{2}\right)+\Gamma\left(\sigma^{2}\right) U^{-1} \bar{R}\right) / \mu$ \\
\hline$\phi$ & $\left(\sigma^{2} e^{-\mu U}+e^{-\mu} \bar{R}\right) / U \mu$ & $\left(\bar{R} / \sigma^{2}+\mu U\right) \Gamma\left(\sigma^{2}\right)+\mu U \bar{R}$ & $\exp \left(\sigma^{2}\right)\left(\bar{R} / \sigma^{2}+\Gamma\left(\sigma^{2}\right)\right)+\mu U \bar{R}$ \\
\hline$\varphi$ & $\Gamma(U \mu) \exp \left(\bar{R} / \sigma^{2}+\mu U\right) / \sigma^{2}$ & $\Gamma(U)\left(\exp \left(\bar{R} / \sigma^{2}+\mu U\right)+\Gamma(U \mu)\right)^{-1}$ & $\Gamma(U)\left(\exp \left(\bar{R} / \sigma^{2}+\mu U\right)+\left(\bar{R} / \sigma^{2}+\mu U\right)\right)^{-1}$ \\
\hline \hline
\end{tabular}

$t \rightarrow \infty$. Thus, we should study the difference between $L(\mathcal{P}(t+1))$ and $L(\mathcal{P}(t))$ :

$$
\begin{aligned}
L(\mathcal{P}(t+1))-L(\mathcal{P}(t)) & \\
\quad & \quad \underset{(a)}{\leq} \phi(\mathcal{P}(t+1) / \mathcal{P}(t)) \cdot(\mathcal{P}(t+1)-\mathcal{P}(t)) \\
& \stackrel{(b)}{\leq} \phi(\mathcal{P}(t)) \cdot(\mathcal{P}(t+1)-\mathcal{P}(t)),
\end{aligned}
$$

where $(a)$ follows from the definition of $G$ and $(b)$ comes from the fact that $f$ is 1-Lipschitz ${ }^{2}$. Therefore, we have (10), where $(c)$ follows from the fact that $\phi(\mathcal{P}(t))$ is 1-Lipschitz.

Step 3: Approximating the optimal bound of $\mathbb{E}[W(\mathcal{P})]$.

Recall that the mobility velocity $V_{u}$ has finite mean $\mu$ and variance $\sigma^{2}$, from the previous steps, $\omega_{\text {avg }}(0)$ is denoted by

$$
\omega_{a v g}(0) \propto \Theta\left(U^{\frac{-\sigma^{2}}{\bar{R} U \mu}}\right) .
$$

Combining with Lemma 1, we have

$$
\mathbb{E}\left[\phi(\mathcal{P}(0)) \cdot \omega_{\text {avg }}(0)\right] \leq \Theta\left(\exp \left(-\frac{\left(\sigma^{2}+1\right)}{\bar{R} U \mu}\right)\right),
$$

in particular, the equation operation is achieved when the functions in Table 1 are employed. Therefore, we have

$$
\mathbb{E}[W(\mathcal{P})] \leq U \cdot \mathbb{E}\left[\phi(\mathcal{P}(0)) \cdot \omega_{\text {avg }}(0)\right],
$$

which completes the proof of Theorem 1.

\subsection{Asymptotical Convergence Rate}

In terms of the ACR of QAMCS, it is clear that the key point is to study the convergence of $\omega_{u, f} P_{u, f}$.

Lemma 2. For each device $u, \omega_{u, f} P_{u, f}$ converges to a steady state, that is

$$
\mathbb{P}\left(\lim _{t \rightarrow \infty} \omega_{u, j}(t) P_{u, f}(t) \rightarrow \phi\left(\omega_{u, j}(0) P_{u, f}(0)\right)\right)=1,
$$

where the function $\phi(x)$ is defined in Table 1.

To prove Lemma 2, we first investigate the relationship between $\omega_{u, j}(t)$ and $\omega_{a v g}(0)$.

Proposition 1. For any device $u$ and $j$, we have

$$
\lim _{t \rightarrow \infty} \sum_{u} \sum_{j}\left\|\omega_{u, j}(t)\right\| / U^{2} \rightarrow \omega_{a v g}(0) .
$$

${ }^{2}$ A function $\phi: \mathbb{R} \rightarrow \mathbb{R}$ is 1-Lipschitz if $\phi\left(t_{1}\right)-\phi\left(t_{2}\right) \leq$ $\left|t_{1}-t_{2}\right|$ for all $t_{1}, t_{2} \in \mathbb{R}$.
Proof. We use the tool of matrix Frobenius norm, in which the convergence of the sample covariances is able to approximate the true covariance. Hence, there exists a finite constant $V_{r}\left(V_{r}<\infty\right)$, such that for each device $u$,

$$
\sup _{t \geq 0} \sum_{u}\left\|\overline{P_{u}}(t)\left(P_{u}(t)+\max _{u} P_{u}(t)\right)\right\| / U \leq V_{r},
$$

where $P_{u}(t)=\sum_{f} P_{u, f}(t)$ and $\overline{P_{u}}(t)=\sum_{u=1}^{U} P_{u}(t) / U$. Let $\omega_{u}(t)=\max _{j} \omega_{u, j}(t)-\omega_{\text {avg }}(t)$ denote the deviation of the information estimation for device $u$ from $\omega_{\text {avg }}(t)$. Also, let $\underline{\omega_{u}}$ and $A_{u}$ respectively denote the matrices $\left[\underline{\omega_{u}(t)}\right]_{U}$ and $\left[A_{u}(t)\right]_{U}$, where $A_{u}(t)=\left(P_{u}(t)+\phi\left(P_{u}(t)\right)\right)^{-1}$. Due to the Markov process of the D2D network, we have

$$
\begin{gathered}
\underline{\omega_{u}(t+1)}=\left(\overline{P_{u}}(t)-\omega_{u}(t) \otimes P_{u}(t)\right) \underline{\omega_{u}(t)} \\
+\left(A_{u}-A_{\text {avg }}(t)\right),
\end{gathered}
$$

where $A_{\text {avg }}(t)=\frac{1}{N} \sum_{i=1}^{N} A_{u}(t)$. Note that, by (13), there exists a bounded $\left\{G_{u}(t)\right\}$, which is defined in (7). By $[9$, Proposition 1], there exists a $[0,1]$-valued $\left\{G_{u}(t+1)\right\}$ adapted process $\underline{\underline{\omega_{u, j}}}(j \neq u)$, such that,

$$
\left\|\overline{P_{u}}(t)-\omega_{u}(t) \otimes P_{u}(t)\right\| \leq \sum_{j \neq u}\left(1-\underline{\underline{\omega_{u, j}}}\right) \max _{t}\left\|\underline{\omega_{u}(t)}\right\| .
$$

Thus, we have

$$
\begin{aligned}
& \left\|\overline{P_{j}}(t)-\overline{P_{j}}(t) \otimes P_{u}(t)\right\|^{2} \leq \\
& \quad \sum_{j}\left(1-\underline{\underline{\omega_{u, j}}}\right)^{2} \max _{t}\left\|\underline{\omega_{u}(t)}\right\|^{2} \leq\left(1-\underline{\underline{\omega_{u}}}\right)^{2} \max _{t}\left\|\underline{\omega_{u}(t)}\right\|^{2},
\end{aligned}
$$

where $\underline{\omega_{u}}$ is the $\left\{G_{u}(t+1)\right\}$ adapted process. By the conditional $\overline{\overline{J e n s e n}}$ 's inequality, we have

$$
\mathbb{E}\left[\max _{t}\left\|\underline{\omega_{u}(t)}\right\| \mid G_{u}(t)\right] \geq \wedge_{\mathrm{j}=1}^{U} \mathbb{E}\left[\underline{\underline{\omega_{u, j}}} \mid G_{u}(t)\right] .
$$

Therefore, we can complete the proof of Proposition 1.

Based on Proposition 1, we can have the convergence of $\left\{\omega_{\text {avg }}(t)\right\}$.

Proposition 2. For each device $u$, we have

$$
\lim _{t \rightarrow \infty} \omega_{\text {avg }}(t) \rightarrow G_{u}(0) \otimes P_{u}(0) .
$$

Proof. In fact, $\omega_{\text {avg }}(t)$ satisfies

$$
\omega_{a v g}(t+1)=\left(1-\frac{A_{a v g}(t)}{\max _{u} A_{u}(t)}\right) \omega_{a v g}(t)+\frac{A_{a v g}(t)}{\max _{u} A_{u}(t)} .
$$




$$
\begin{aligned}
L(\mathcal{P}(t+1)) & -L(\mathcal{P}(t)) \leq(\Phi(\mathcal{P}(t+1))-\Phi(\mathcal{P}(t))) \cdot O\left(\max _{j} \omega_{u, j} P_{u, f}\right) \\
& \leq \phi(\mathcal{P}(t)) \cdot\left((\Phi(\mathcal{P}(t+1))-\Phi(\mathcal{P}(t)))-\int_{t}^{t+1} \mathcal{P}(y) \cdot \mathbf{1}_{\{\mathcal{P}(y)>\pi(y)\}} d y\right) \\
& \stackrel{(c)}{\leq} \phi(\mathcal{P}(t+1)-\mathcal{P}(t)) \cdot(\Phi(\mathcal{P}(t+1))-\Phi(\mathcal{P}(t)))-\int_{t}^{t+1} \phi(\mathcal{P}(y)) \mathcal{P}(y+1) / \mathcal{P}(y) \cdot \mathbf{1}_{\{\mathcal{P}(y)>\pi(y)\}} d y \\
& \leq \phi(\mathcal{P}(t+1)-\mathcal{P}(t)) \cdot(\Phi(\mathcal{P}(t+1))-\Phi(\mathcal{P}(t)))-\int_{t}^{t+1} \phi(\mathcal{P}(y)) \mathcal{P}(y) d y
\end{aligned}
$$

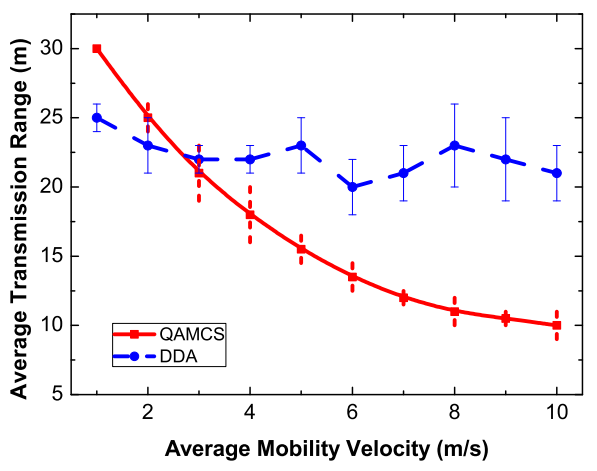

Figure 2: $\bar{R}$ comparison when $U=40$.

Let $\overline{\omega_{\text {avg }}(t)}$ denote the residual

$$
\omega_{a v g}(t)-G_{u}(0) \otimes P_{u}(0),
$$

and the process $\overline{\omega_{\text {avg }}(t)}$ satisfies:

$$
\begin{aligned}
& \overline{\omega_{\text {avg }}(t+1)}=\left(1-\frac{A_{\text {avg }}(t)}{\max _{u} A_{u}(t)}\right) \overline{\omega_{\text {avg }}(t)}+ \\
& \left(\omega_{\text {avg }}(t)-G_{u}(0) \otimes P_{u}(0)\right) \frac{A_{a v g}(t)}{\max _{u} A_{u}(t)} .
\end{aligned}
$$

We can complete the proof immediately.

According to the results of Proposition 1 and Proposition 2, it is straightforward to complete the proof of Lemma 2. Therefore, the results the Theorem 2 can be obtained immediately.

\section{NUMERICAL RESULTS}

In this section, we evaluate the performance of QAMCS using extensive simulations and validate our theoretical analysis. Specifically, multiple devices with total 30 applications move within a cellular network of $5 \mathrm{~km} \times 5 \mathrm{~km}$. The size of each user's cache is 10 applications. In this work, we assume that the size of the application is the same. The initial transmission radius and mobility velocity are $R_{u}=30 \mathrm{~m}$ and $V_{u}=5 \mathrm{~m} / \mathrm{s}$, respectively. To reveal the performance improvements, we compare QAMCS with two alternative schemes.

- Coordinated dissemination algorithm (CDA). In this case, the BS acts as the central controller, which implements the application dissemination, cache update, and transmission fashion, assuming that the popularity of each application and the corresponding application distribution of each cache are known.
- Direct dissemination algorithm (DDA). A moving device carries a packet until a new device moves into its transmission range, and then, it forwards the application [10]. For fair comparison, we also apply the D2D time function and priority function to DDA.

At first, we evaluate the performance of QAMCS by assuming the number of devices, $U$, is a constant. Fig. 1(a) and Fig. 1(b) show the total user satisfaction along with time at different mobility velocities. From the given results, it is obvious that QAMCS can achieve a better performance than DDA and is comparable to the optimal solution CDA, in particular, the average user satisfaction using the proposed QAMCS is very close to that of CDA when the distributed algorithm converges. Moreover, we can also observe that the mobility is indeed beneficial to the user satisfaction, that is because high mobility can reduce the communication cost by lowing the transmission range, which can be explicitly obtained from Fig. 2.

Next, we validate the performance of QAMCS in a dynamic environment, in which users may randomly join and leave the networks. In Fig. 3(a), we plot the corresponding total user satisfaction of all the competing schemes. It is clear that the dynamic environment indeed impacts the performance of QAMCS. However, fortunately, the proposed QAMC$\mathrm{S}$ is still applicable to a dynamic environment. Moreover, Fig. 3(b) exhibits the number of iteration of the QAMCS in different kinds of dynamic environment, which can be viewed as a picture proof of Theorem 2. Therefore, the above results confirm that the proposed QAMCS also works well in a dynamic environment.

\section{CONCLUSIONS}

This paper is devoted to designing a QoE-aware data dissemination scheme for a practical mobile device-to-device communication system. Complementary to the previous works, we advocate the method of distributed data dissemination to shed new light on traditionally challenging issues on system heterogeneity. Specifically, we investigate the relationship between the mobility and performance based on observed data popularity and priority from each device. We further provide a general performance property bound for any distributed scheme. Importantly, by dynamically setting the transmission range, D2D time, and transmission fashion, we design a class of distributed scheme to achieve the optimal performance in a fully distributed manner.

\section{Acknowledgements}

This work is partly supported by the State Key Development Program of Basic Research of China (2013CB329005), by the National Natural Science Foundation of China (Grant No. 61322104, No. 61201165, and No. 61271240), and by the 


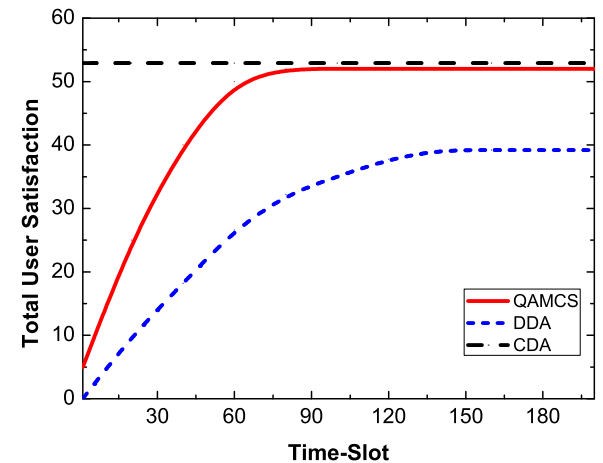

(a) $\bar{V}=5 \mathrm{~m} / \mathrm{s}$

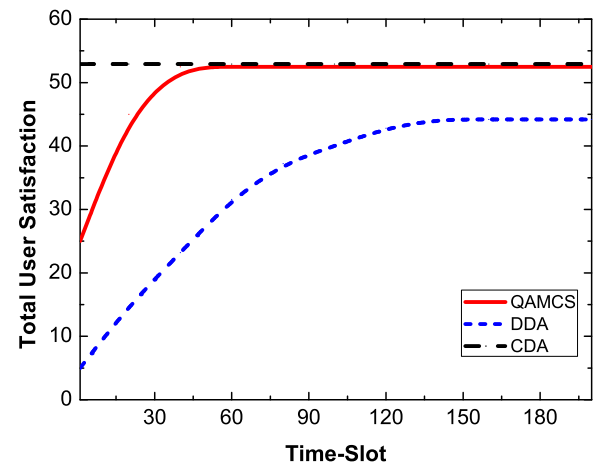

(b) $\bar{V}=10 \mathrm{~m} / \mathrm{s}$

Figure 1: Performance comparison in terms of total user satisfaction when $U=40$.

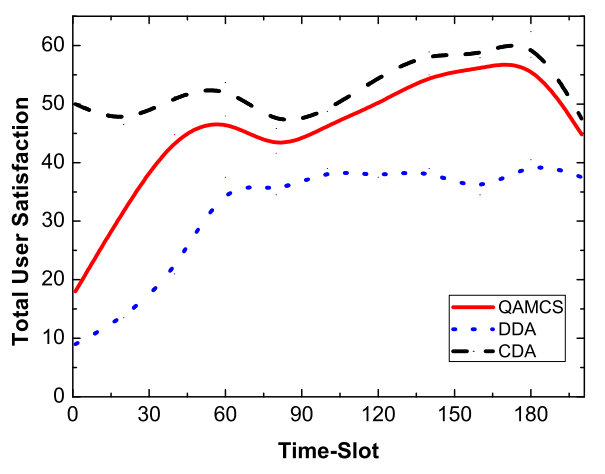

(a) $20 \leq U \leq 60$

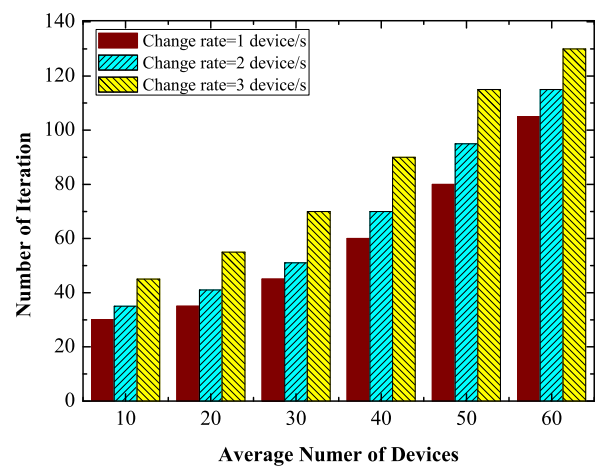

(b) ACR

Figure 3: Performance comparison in a dynamic environment. 
Priority Academic Program Development of Jiangsu Higher Education Institutions.

\section{REFERENCES}

[1] G. Fodor, E. Dahlman, G. Mildh, S. Parkvall, N. Reider, G. Miklos, and Z. Turanyi, "Design Aspects of Network Assisted Device-to-Device Communications,"IEEE Communications Magazine, vol. 50, no. 3, pp. 170-177, 2012.

[2] D. Wu, J. Wang, R. Q. Hu, Y. Cai, "Energy-Efficient Resource Sharing for Mobile Device-to-Device Multimedia Communications," IEEE Transactions on Vehicular Technology, vol. 63, no. 5, pp. 2093-2103, Jun. 2014.

[3] Y. Li, Z. Wang, D. Jin, S. Chen, "Optimal Mobile Content Downloading in Device-to-Device Communication Underlaying Cellular Networks," IEEE Transactions on Wireless Communications, vol. 13, no. 7, pp. 3596-3608, July 2014.

[4] L. Zhou, Y. Wen, H. Wang, and M. Guizani, "Resource Allocation with Incomplete Information for QoE-driven Multimedia Communications," IEEE Transactions on Wireless Communications, vol. 12, no. 8, pp. 3733-3745, 2013.

[5] L. Zhou, Y. Zhang, K. Song, W. Jing, and A. Vasilakos, "Distributed Media-Service Scheme for P2P-based Vehicular Networks," IEEE Transactions on Vehicular Technology, vol. 60, no. 2, pp. 692-703, 2011.

[6] Y. Zhu, L. Song, S. Wu, H. Wang, C. Wang, "Cooperative Stepwise Relaying and Combining for Multi-hop Vehicular Wireless Communication," to appear in IEEE Transaction on Vechicular Technology.

[7] S. Shakkottai, R. Johari, "Demand-aware content distribution on the internet," IEEE/ACM Transactions on Networking, vol. 18, no. 2, 476-489, April 2010.

[8] J. Reich and A. Chaintreau, "The age of impatience: Optimal replication schemes for opportunistic networks," in Proc. ACM CoNEXT, 2009.

[9] L. Zhou, H.-H. Chen, "On Distributed Multimedia Scheduling with Constrained Control Channels," IEEE Transactions on Multimedia, vol. 13, no. 5, pp. 1040-1051, 2011.

[10] J. Zhao and G. Cao, "VADD: Vehicle-assisted data delivery in vehicular ad hoc networks," IEEE Trans. Veh. Technol., vol. 57, no. 3, pp. 1910-1922, 2008. 